\title{
Time resolved particle image velocimetry experimental study of the near wake characteristics of a horizontal axis wind turbine*
}

\author{
Jian-wen $\mathrm{WANG}^{1,3}$, Ren-yu YUAN ${ }^{2}$, Xue-qing DONG ${ }^{1,3}$, San-xia ZHANG ${ }^{2}$, Yang $\mathrm{SONG}^{1,3}$, \\ Zhi-ying $\mathrm{GAO}^{1,3}$, Kun LUO ${ }^{1,2}$, Kun-zan QIU ${ }^{\dagger ‡ 2}$, Ming-jiang $\mathrm{NI}^{2}$, Ke-fa CEN ${ }^{2}$ \\ ( ${ }^{1}$ School of Energy and Power Engineering, Inner Mongolia University of Technology, Huhhot 010051, China) \\ ('State Key Laboratory of Clean Energy Utilization, Zhejiang University, Hangzhou 310027, China) \\ $\left({ }^{3}\right.$ Key Laboratory of Wind and Solar Power Energy Utilization Technology Ministry of Education and Inner Mongolia Construction, \\ Huhhot 010051, China) \\ ${ }^{\dagger}$ E-mail: qiukz@zju.edu.cn
}

Received Oct. 30, 2014; Revision accepted Apr. 9, 2015; Crosschecked June 16, 2015

\begin{abstract}
Wind tunnel experiments at a model scale have been carried out to investigate the flow characteristics in the near wake of a wind turbine. Time resolved particle image velocimetry (TRPIV) measurements are applied to visualize the wind turbine wake flow. The instantaneous vorticity, average velocities, turbulence kinetic energy, and Reynolds stresses in the near wake have been measured when the wind turbine is operated at tip speed ratios (TSRs) in the range of 3-5. It was found that wind turbine near the wake flow field can be divided into a velocity increased region, a velocity unchanged region, and a velocity deficit region in the radial direction, and the axial average velocities at different TSRs in the wake reach inflow velocity almost at the same radial location. The rotor wake turbulent kinetic energy appears in two peaks at approximately $0.3 R$ and $0.9 R$ regions in the radial direction. The Reynolds shear stress is less than the Reynolds normal stresses, the axial Reynolds normal stress is larger than the Reynolds shear stress and radial Reynolds normal stress in the blade root region, while the radial Reynolds normal stress is larger than the Reynolds shear stress and axial Reynolds normal stress in the blade tip region. The experimental data may also serve as a benchmark for validation of relevant computational fluid dynamics (CFD) models.
\end{abstract}

Key words: Time resolved particle image velocimetry (TRPIV), Wind turbine, Tip speed ratio (TSR), Near wake, Flow characteristics

doi:10.1631/jzus.A1400332

Document code: A

CLC number: TK83

\section{Introduction}

With the fast growing number of wind farms being installed worldwide, it is necessary to obtain a deep understanding of wind turbine working principles and its energy conversion process. Today, wind turbines are often placed close together in small clus-

\footnotetext{
Corresponding author

* Project supported by the National Natural Science Foundation of China (No. 51366010), and the Inner Mongolia Autonomous Region Open Major Basic Research Project (No. 20120905), China

(iD ORCID: Jian-wen WANG, http://orcid.org/0000-0001-7420-5322; Kun-zan QIU, http://orcid.org/0000-0003-0978-2697

(C) Zhejiang University and Springer-Verlag Berlin Heidelberg 2015
}

ters or in large wind farms (Ivanell et al., 2009), which means that they operate in the wakes of the upstream wind turbines. Inflow conditions dominated by vortical structures created by upstream turbines may reduce the power performance for the downstream turbines. Thus, a good understanding and modeling of the wake behavior of wind turbines is of great significance to guide the operations of wind farms.

Wind turbine wakes can be distinctly divided into the near wake regions and the far wake regions. The near wake region is taken as the area just behind the rotor, approximately up to one rotor diameter downstream (Vermeer et al., 2003). The wind turbine near the wake region is characterized by a complex 
coupled vortex system, unsteadiness, and strong turbulence heterogeneity (Zhang et al., 2012). The investigation of wind turbine's near wake flow characteristics has important significations. It constitutes the basis of wind turbine load calculation, aeroacoustic noise control, and blade design. As the numerical simulation model does not yet have sufficient accuracy, the experimental method to explore the mechanism of wind turbine wake flow is still of great significance. In the past few years, there have been many investigations on the wind turbine near wake which primarily focus on their global properties, such as power coefficient $C_{\mathrm{p}}$ (Jiang et al., 2007; Chu and Chiang, 2014), axial force coefficient $C_{\mathrm{T}}$, average velocities (Vermeer, 2001; Abdelsalam et al., 2014), and tip vortex properties (Whale and Anderson, 1993; Lignarolo et al., 2014). The investigation of the distribution of average velocity behind the horizontalaxis wind turbine rotor has revealed an expansion and a decay of the 3D wake (Hu and $\mathrm{Du}, 2009)$. Some experimental investigations into the properties of the vortex wake behind a wind turbine rotor have revealed the formation and evolution of the helical tip vortices (Whale et al., 2000; Yang et al., 2012). It can be found that the previous studies on the wake tend to either concern velocity distribution or tip vortex properties, and only a few studies have been done on turbulent kinetic energy and Reynolds stresses in the near wake. However, turbulent flow characteristics reflected by turbulent kinetic energy and Reynolds stresses have a significant impact on the energy output process of wind turbines (Breton et al., 2014). Therefore, in this paper, the wind turbine near wake flow parameters, including instantaneous vorticity, average velocities, turbulent kinetic energy, and Reynolds stresses, are studied, and their evolution rules in the near wake are summarized.

Phase-locked particle image velocimetry (PIV) is now widely employed to measure flow statistics such as velocity (Hirahara et al., 2005; Lee and Wu, 2013), turbulence intensity (Whale et al., 1996; Liu et al., 2010), shear stress (Angele and MuhammadKlingmann, 2006; Stafford et al., 2012), and vorticity (Whale et al., 2000; Jin et al., 2014), and to subsequently identify large-scale coherent flow structures. Compared with traditional PIV, time resolved particle image velocimetry (TRPIV) has a higher sampling frequency and can grasp more detailed information of flow structures. TRPIV possesses obvious advantages in measuring the instantaneous flow field parameters, so it will play a more important role in the investigation of wind turbine wakes. In our present experiment, TRPIV is applied to measure wind turbine flow properties at different tip speed ratios (TSRs).

The present paper addresses the need for detailed measurements of wind turbine near wakes, to establish an experimental database to validate computational fluid dynamics models. Both instantaneous properties and average properties are presented, and the flow properties are compared among different TSRs. All the flow properties and the size are normalized, so the results can also serve as a reference to the experimental or simulated data of different operating conditions.

\section{Experimental setup}

The experimental setup is composed of a wind turbine model, closed wind tunnels, TRPIV testing system, and a tracer particle generator. Fig. 1 shows the overall layout of the experimental equipment. Fig. 2 shows the size of the wind tunnel, the wind tunnel cross-sectional area, which is a square whose size is $920 \mathrm{~mm} \times 920 \mathrm{~mm}$, and a wind turbine main shaft with a center distance from the ground of $460 \mathrm{~mm}$, and the radius of the wind turbine blade is $165 \mathrm{~mm}$. The blockage ratio of the rotor swept area to the cross-sectional area of the wind tunnel in the present experiment is $10.1 \%$. According to previous studies (Chen and Liou, 2011; Chu and Chiang, 2014), the blockage correction is less than $5 \%$ and not required when the blockage ratio is around $10 \%$ in the wind tunnel experiment. Therefore, the influence of the blockage ratio is limited and no blockage correction is performed in the present study. Fig. 3 shows the testing window for the TRPIV experiment. The wind turbine model used in the experiment is a miniature of a $100 \mathrm{~W}$ wind turbine, and the size ratio of the model to the wind turbine prototype is 1:4.2. The rated wind speed of the $100 \mathrm{~W}$ wind turbine is $8 \mathrm{~m} / \mathrm{s}$, the starting wind speed is $3.5 \mathrm{~m} / \mathrm{s}$, and the rated TSR is 5 . The NACA4415 airfoil is adopted in the wind turbine model, and the detailed chord and twist distributions of the blade are shown in Table 1.

The experiments were conducted in the Key Laboratory of the Wind and Solar Power Energy Utilization Technology Ministry of Education and Inner Mongolia Construction, China. The wind turbine was arranged at $\mathrm{B} 1 / \mathrm{K} 2$ closed test section in the 


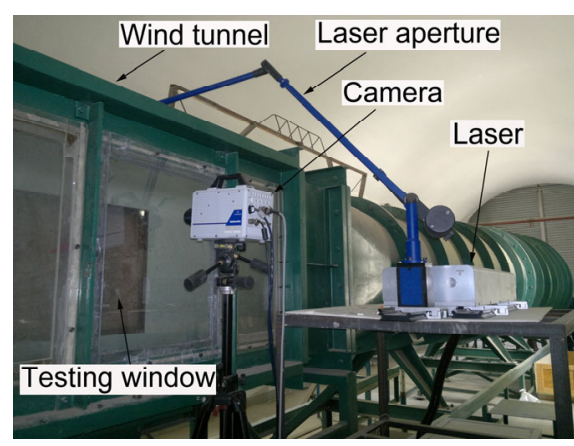

Fig. 1 Experimental setup

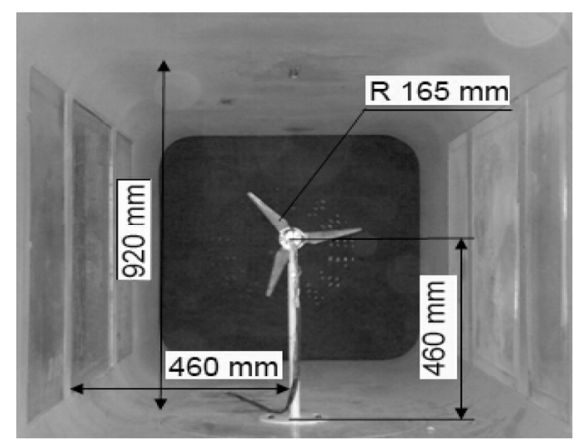

Fig. 2 Diagram of the wind tunnel size

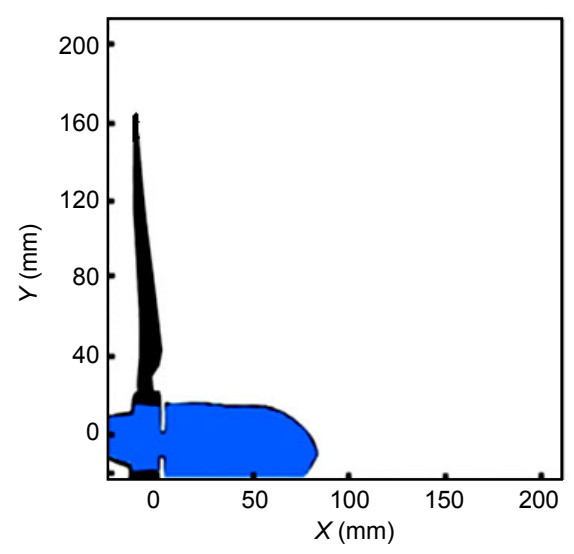

Fig. 3 TRPIV testing window

Table 1 Blade chord and twist distributions in the radial direction

\begin{tabular}{ccc}
\hline Radial distance $(\mathrm{m})$ & Chord length $(\mathrm{m})$ & Twist $\left({ }^{\circ}\right)$ \\
\hline 0.024 & 0.0140 & 0 \\
0.037 & 0.0311 & 23.3 \\
0.053 & 0.0280 & 20.1 \\
0.069 & 0.0249 & 17.0 \\
0.085 & 0.0219 & 13.8 \\
0.101 & 0.0190 & 10.6 \\
0.117 & 0.0161 & 7.40 \\
0.133 & 0.0132 & 4.20 \\
0.149 & 0.0103 & 1.00 \\
0.165 & 0.0073 & -2.20 \\
\hline
\end{tabular}

wind tunnel. The wind speed can be adjusted in the range of $0-60 \mathrm{~m} / \mathrm{s}$, and the experiments are conducted at chord Reynolds numbers in the range of 5455 -23145. The TRPIV test system uses the Nd:YLF laser (German LaVision Company) with an operating frequency range of $1-10 \mathrm{kHz}$. The peak energy of the laser is $200 \mathrm{~mJ}$, while the energy is $10 \mathrm{~mJ}$ when the frequency is $1 \mathrm{kHz}$, and the pulse duration is $150 \mathrm{~ns}$. The maximum frame rate of high-speed 12 bit charge-coupled device (CCD) camera can be up to $5400 \mathrm{fps}$, and the frame rate in this experiment is set at $1000 \mathrm{fps}$, while the corresponding resolution is $1024 \times 1024$ pixels. Tracer particles in these experiments are using dioctyl sebacate (DEHS), and its particle diameter is about $1 \mu \mathrm{m}$.

The PIV data and the mode for the correlation can be selected by using the vector calculation. The cross-correlation algorithm is applied to calculate a vector field on two single-exposed images. The PIV evaluation is performed in a multi pass with decreasingly smaller window sizes, and the window sizes are between $32 \times 32$ pixels and $64 \times 64$ pixels with $75 \%$ and $50 \%$ overlaps, respectively. In addition, the high accuracy mode for the final passes is applied so that a more sophisticated reconstruction algorithm can be used for the image correction and reconstruction.

The wind turbine yaw angle is $0^{\circ}$ in the present experiments, and the laser beam is vertical to the plane of the ground and goes through the rotation axis of the wind wheel. The acquisition camera is placed outside the wind tunnel and is vertical to the laser plane. Through the organic glass window, the filming area of the camera is about $230 \mathrm{~mm} \times 230 \mathrm{~mm}$. One of the wind turbine blades is defined as the reference No. 1 blade and the plane where the reference No. 1 blade leading edge is rotated to the laser plane as the $0^{\circ}$ plane. Using the phase locked position system, data collection begins when the No. 1 blade rotates to the $0^{\circ}$ plane, and 1000 samples are collected every second as the sampling frequency is $1000 \mathrm{~Hz}$. In the present experiments, the inflow wind velocity is $11 \mathrm{~m} / \mathrm{s}$, the turbulence intensity of inflow is $0.5 \%$, and the TSRs are in the range of 3-5. The testing conditions are summarized in Table 2.

Table 2 Testing conditions set

\begin{tabular}{ccc}
\hline TSR & Blade tip speed (m/s) & Wheel rotation speed (r/min) \\
\hline 3 & 33 & 1911 \\
4 & 44 & 2548 \\
5 & 55 & 3185 \\
\hline
\end{tabular}




\section{Results and discussion}

\subsection{Evolution of instantaneous flow structures}

With the help of TRPIV technology, instantaneous flow field data can be collected at a high frequency. To analyze the evolution rules of near wake instantaneous flow structures, the flow parameters are measured every $0.001 \mathrm{~s}$ in our present study. Fig. 4 shows the instantaneous vortex structures when the TSR is 4 and the No. 1 blade rotates to the $0^{\circ}$ plane. The process of vortex generation and shedding in the near wake can be observed from Fig. 4. In addition, unsteady vortex structures shedding from the blade roots and turbine nacelle are also visualized clearly from the PIV measurement results.

The formation and evolution rules of the wind turbine near the wake vortex structures are displayed in Fig. 5, which shows the near wake vortex from $0 \mathrm{~s}$

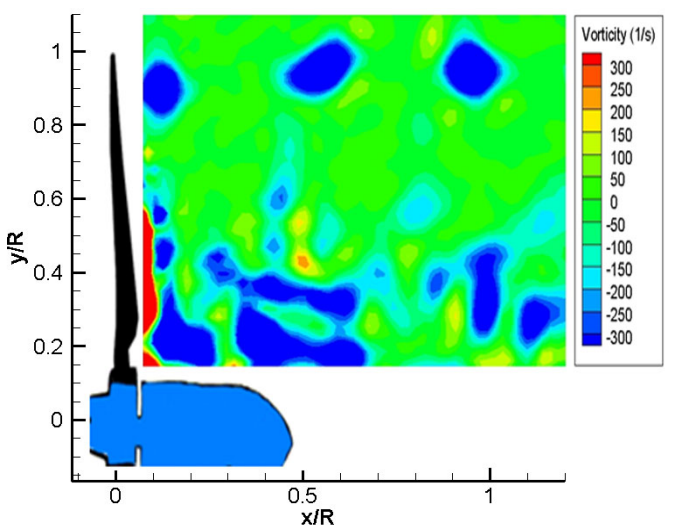

Fig. 4 Instantaneous vortex structures when tip speed ratio is four
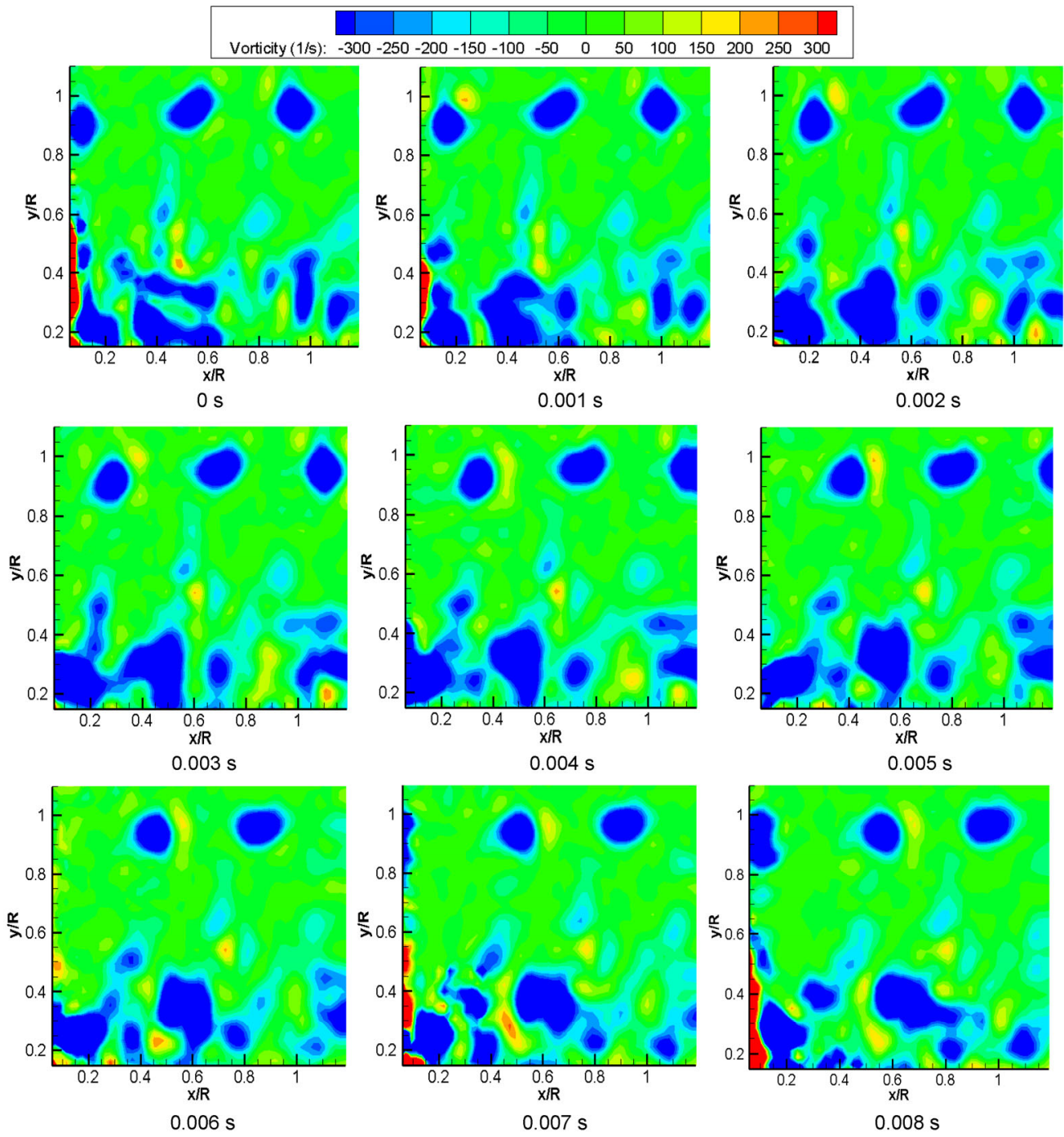

Fig. 5 Evolution of instantaneous vortex structures when the tip speed ratio is four 
to $0.008 \mathrm{~s}$ as the time that the wind turbine rotates $1 / 3$ circular is $0.00785 \mathrm{~s}$ when the TSR is 4 . It was found that a blade tip vortex generated when No. 1 blade rotates to $0^{\circ}$ plane at $0 \mathrm{~s}$, and then the tip vortex sheds from the turbine blade and moves downstream. As shown in Fig. 4, the blade tip vortex induced by the No. 1 blade and the other two tip vortices induced by other blades form a moving tip vortex array in the $0^{\circ}$ plane. The next blade tip vortex generation can be observed at $0.008 \mathrm{~s}$ (about $1 / 3$ rotor rotation period).

\subsection{Average velocities}

Fig. 6 shows the contours of a normalized axial average velocity $u / U$ when the inflow wind velocity $U$ ( $U$ refers to the inflow velocity at the turbine hub height) is $11 \mathrm{~m} / \mathrm{s}$ and the TSR is 4 . This result displays part of the flow areas behind the wind turbine, and the position of the wind turbine can be seen in Fig. 6 . The obvious velocity increased region and velocity deficit region can be observed, and the velocity deficit region corresponding to the region where the kinetic energy from the wind is transformed into the mechanical energy of the wind turbine rotors. Velocity backflow phenomenon can be observed in the wind turbine blade root region, which is due to the wind energy encountering a great loss of dynamic pressure in this region.

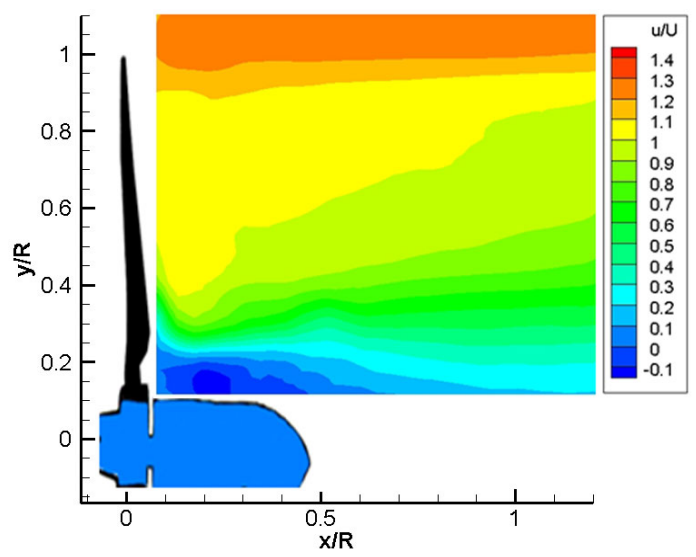

Fig. 6 Contours of normalized axial average velocity $u / U$ when tip speed ratio is four

Detailed flow field information should be obtained, so that the experimental results can be employed to verify the numerical model. Fig. 7 shows the near wake axial average velocity quantitative characteristics of different TSRs at different downstream locations. From these results, it is found that the axial velocity deficit region primarily occurred at $y / R$ less than 0.4 , in the upper wake when $y / R$ is larger than 0.9 , the axial velocity may exceed the inflow wind velocity, and $u / U$ may reach as large as 1.2 because of the blockage effect. In the middle, there exists a region where the velocity nearly remains at the inflow velocity, when $y / R$ is between 0.5 and 0.9 . A conclusion also can be made that the wake influence region in the axial direction is far more than one radius length as the fluid axial average velocity has not fully recovered to the inflow velocity when $x / R=1.0$. Generally speaking, the average velocity shares similar trends for cases with different TSRs, and when $y / R$ is larger than 0.4 , the axial velocity increases as the TSR increases. A phenomenon can be observed in Fig. 7 that the velocity curves at different TSRs converge at one point where $y / R$ is about 0.9 and $u / U$ is about 1.0 , which means that the axial average velocity at different TSRs in the wake reach the inflow velocity almost at the same radial location. As the axial flow interference factor is defined as $a=1-u / U$, the distribution characteristics of the axial flow interference factor can also be acquired from the results, and it can be found that the axial interference factor decreases along the radial direction. The distribution characteristics of the axial interference factor obtained from the wind tunnel experiment can be applied to analyzing the validity of the assumptions of the blade-element momentum method.

To acquire the evolution rules of the axial average velocity along the downstream direction, vertical profiles of the normalized axial average velocity $u / U$ at $x / R=0.2,0.6,1.0$ when the TSR is 4 are presented in Fig. 8. This result suggests that the axial average velocity increases along the downstream direction when $y / R$ is less than 0.25 , which is due to the axial velocity in the blade root region suffering a greater loss in kinetic energy, then it would obtain from the kinetic energy in the upper fluid in the near wake region. The axial average velocity decreased when $y / R$ is between 0.25 and 1.0 , which can be explained by that fluid kinetic energy in this region, which is partly used to compensate the loss in static pressure, as the static pressure of fluid has suffered a great loss when the wind goes through the wind turbine blades. The decline in the axial velocity behind the wind turbine blades would lead to the fluid volume expansion in the near wake, so the axial average 
velocity in the region, with $y / R$ larger than 1.0 , would increase. Accordingly, a conclusion can be made from the analyses that in the $3 \mathrm{D}$ flow field of wind turbine near wake, wind in the region $y / R$ is between 0.25 and 0.9 and would expand toward both the blade tip region and the blade root region. A conclusion can also be made from the distribution of the velocity that the turbulence intensity of the fluid in the near wake would be strengthened after the wind goes through the wind turbine blade.

Fig. 9 shows the contours of the normalized radial average velocity $v / U$ when the inflow wind velocity $U$ is $11 \mathrm{~m} / \mathrm{s}$ and the TSR is 4 . Fig. 10 shows the vertical profiles of the normalized radial average velocity $v / U$ of different TSRs at different downstream locations. The results suggest that the radial velocity decreases along the flow direction. After the inflow wind flows through the wind turbine, the wind obtains the radial velocity by the reaction force from the blade. The radial velocity decreases gradually as

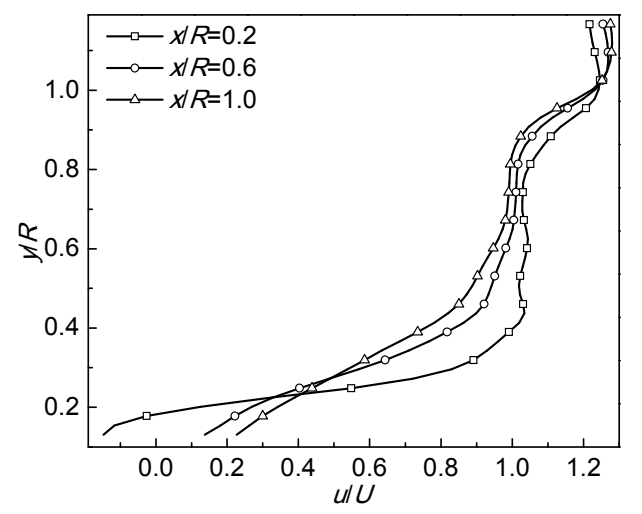

Fig. 8 Vertical profiles of the normalized axial average velocity $u / U$ for three different downstream locations when the tip speed ratio is four the vortex strength decreases along the flow direction. The radial velocity is larger at $y / R=0.4$ in the region above the wind turbine nacelle, which is because this region is affected by the blade root vortex and blade circumferential vortex. At the same TSR, it can be found that the radial velocity has an increased tendency along the radial direction in the later half radial length wake region, and the radial average velocity decreases in the region above the blade tip. When $x / R$ is $0.6,0.8$, and 1.0 , the negative radial velocity can be observed, which signifies that the fluid flows back to the main axis center in these regions.

\subsection{Turbulent kinetic energy}

In a $2 \mathrm{D}$ condition, turbulent kinetic energy can be defined as

$$
\mathrm{TKE}=\frac{1}{2}\left(\overline{u^{\prime 2}}+\overline{v^{\prime 2}}\right)
$$

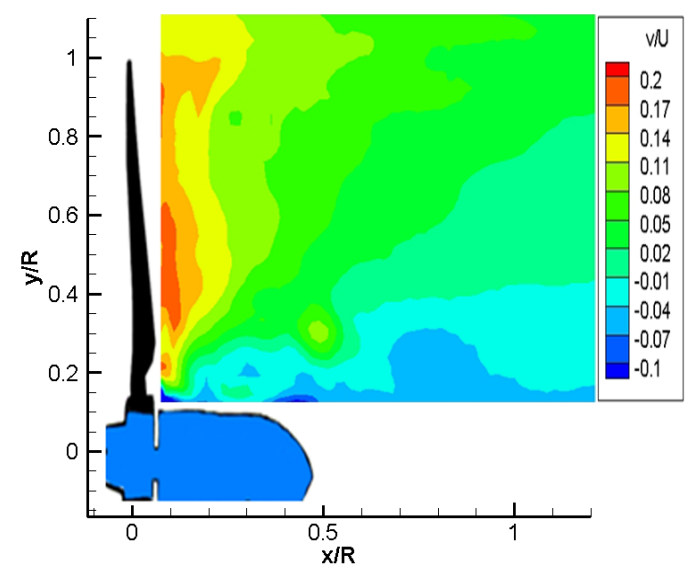

Fig. 9 Contours of normalized radial average velocity $v / U$ when the tip speed ratio is four

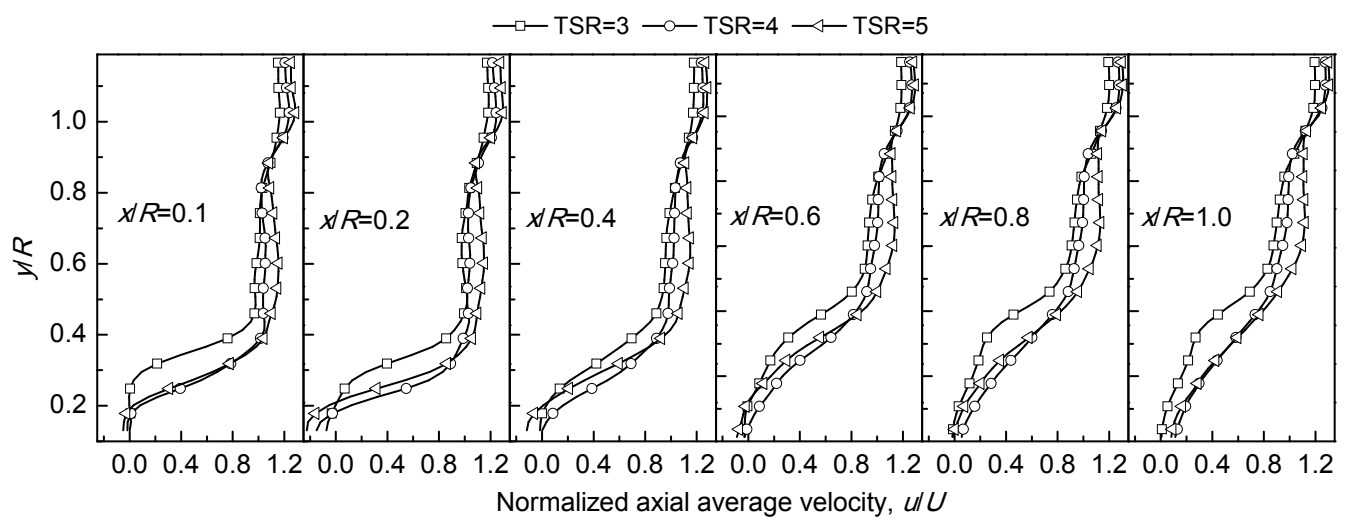

Fig. 7 Vertical profiles of the normalized axial average velocity $u / U$ for three different tip peed ratios at six different downstream locations 
where $u^{\prime}$ denotes the axial velocity fluctuations, and $v^{\prime}$ denotes the radial velocity fluctuations.

Fig. 11 shows the contour of the normalized turbulent kinetic energy when the inflow wind velocity $U$ is $11 \mathrm{~m} / \mathrm{s}$ and the TSR is 4 . Fig. 12 shows the vertical profiles of normalized turbulent kinetic energy of different TSRs at different downstream locations. Generally, turbulent kinetic energy decreases along the flow direction. The turbulent kinetic energy would appear to have two peaks at approximately $y / R=0.3$ and $y / R=0.9$. The turbulent kinetic energy is larger when $y / R=0.2-0.4$, and it also encounters a larger fluctuation among different TSRs in this region. In the region of $y / R=0.6$, the turbulent kinetic energy is smaller than that in both $y / R=0.4$ and $y / R=0.8$, which is a minimum point because both the tip vortices and central vortices have a weaker effect in this region.
The turbulent kinetic energy value in $y / R=0.9$ is larger than that in $y / R=1.0$, which can be explained by the influence of the tip vortex, which primarily focuses on the region $y / R=0.9$ instead of the region $y / R=1.0$.

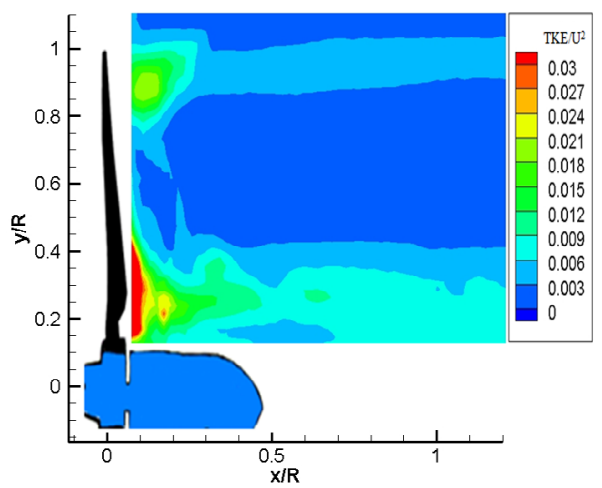

Fig. 11 Contours of normalized turbulent kinetic energy $\mathrm{TKE} / U^{2}$ when the tip speed ratio is four

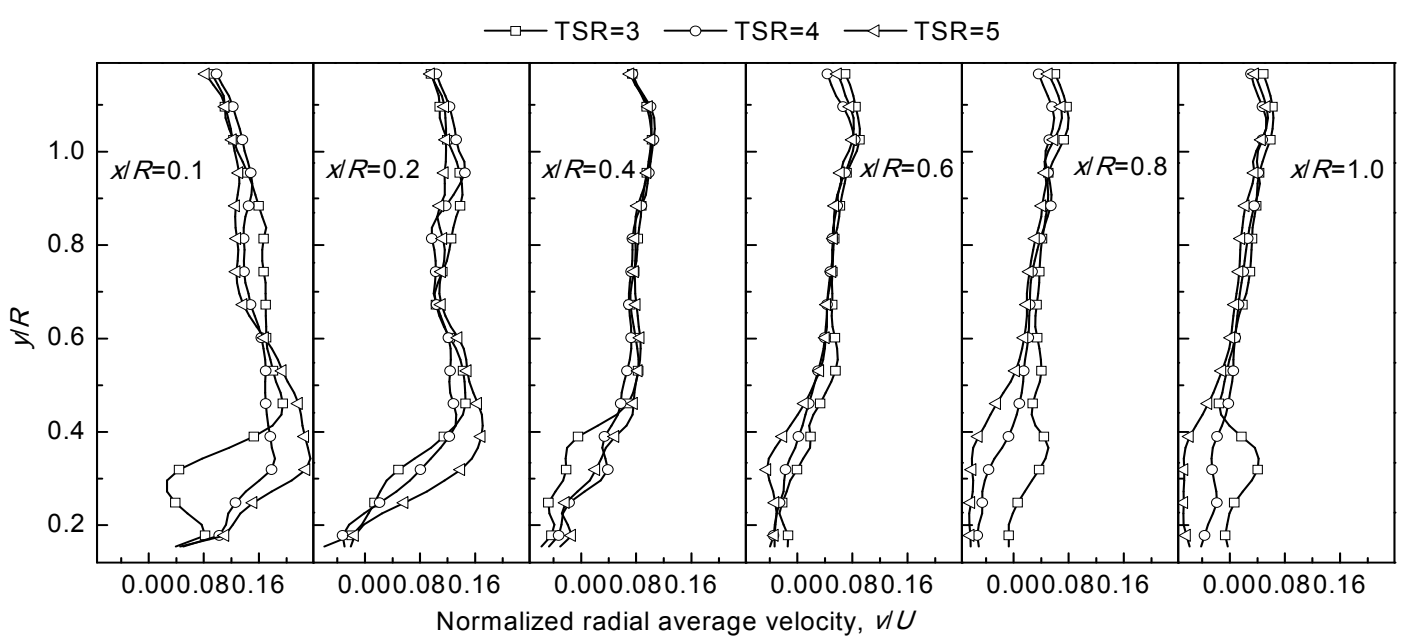

Fig. 10 Vertical profiles of the normalized radial average velocity $v / U$ for three different tip speed ratios at six different downstream locations

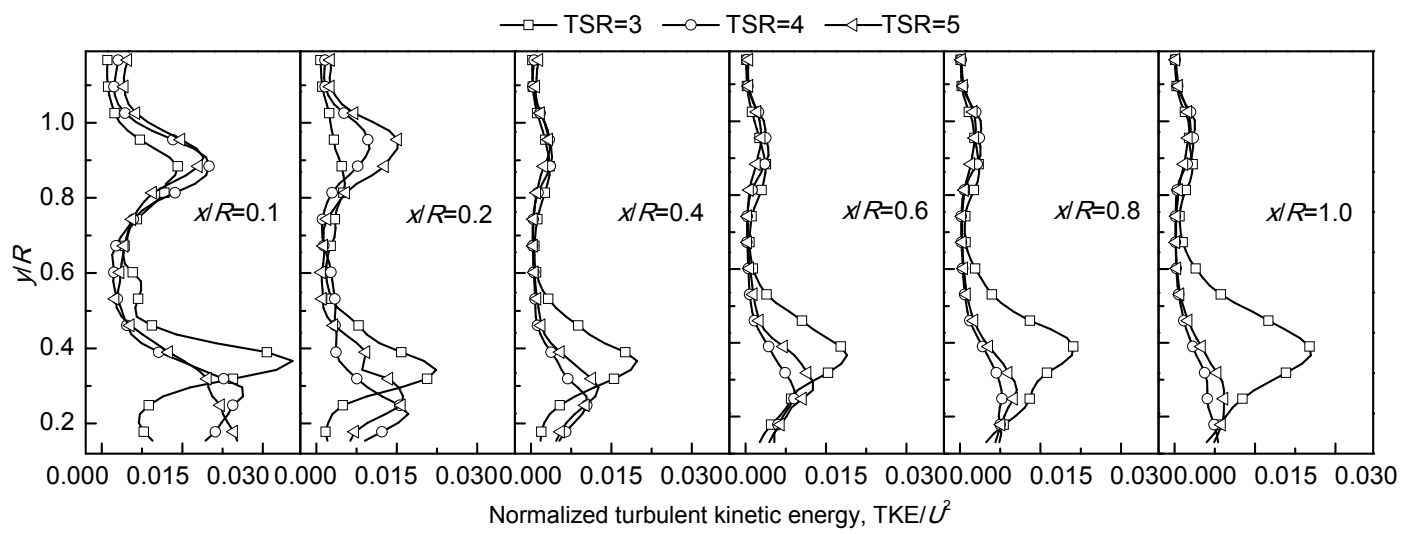

Fig. 12 Vertical profiles of the normalized turbulent kinetic energy TKE/ $U^{2}$ for three different tip speed ratios at six different downstream locations 


\subsection{Reynolds stress}

Fig. 13 shows the contours of the normalized Reynolds stress $\overline{u^{\prime} u^{\prime}} / U^{2}$ when the inflow wind velocity $U$ is $11 \mathrm{~m} / \mathrm{s}$ and the TSR is 4 . Fig. 14 shows the vertical profiles of the normalized Reynolds stress $\overline{u^{\prime} u^{\prime}} / U^{2}$ of different TSRs at different downstream locations. From the results, it can be found that the

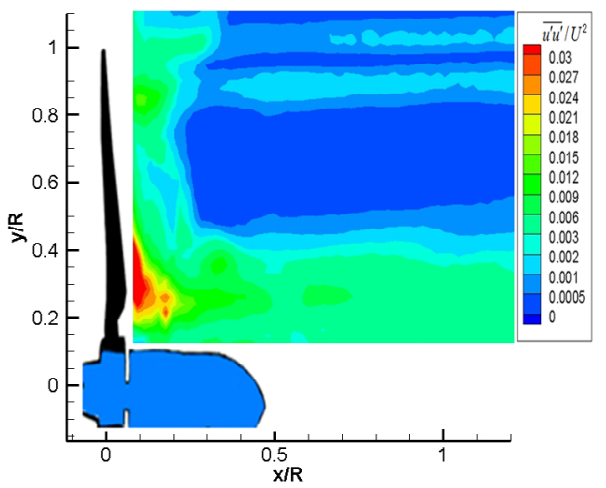

Fig. 13 Contours of the normalized Reynolds stress $\overline{u^{\prime} u^{\prime}} / U^{2}$ when the tip speed ratio is four
Reynolds stress decreases along the axial direction and it goes to zero in the end. The maximum Reynolds stress appears in the region $y / R=0.3-0.4$, which is also the maximum turbulent kinetic energy region. In the region of $y / R=0.6$ and $y / R=1.0$, the Reynolds stress meets its minimum point.

Fig. 15 presents the comparison among different normalized Reynolds normal stresses $\overline{u^{\prime} u^{\prime}} / U^{2}$, $\overline{v^{\prime} v^{\prime}} / U^{2}$ and Reynolds shear stress $\overline{u^{\prime} v^{\prime}} / U^{2}$ when the TSR is 4 . A conclusion can be made from the results that the Reynolds normal stresses and Reynolds shear stress share the same evolution rule, they increased at the blade root and the blade tip due to the effect of the blade root vortices and tip vortices. The Reynolds shear stress $\overline{u^{\prime} v^{\prime}} / U^{2}$ is less than the Reynolds normal stresses $\overline{u^{\prime} u^{\prime}} / U^{2}$ and $\overline{v^{\prime} v^{\prime}} / U^{2}$. In addition, $\overline{u^{\prime} u^{\prime}} / U^{2}$ is larger than $\overline{u^{\prime} v^{\prime}} / U^{2}$ and $\overline{v^{\prime} v^{\prime}} / U^{2}$ in the blade root region, while $\overline{v^{\prime} v^{\prime}} / U^{2}$ is larger than $\overline{u^{\prime} v^{\prime}} / U^{2}$ and $\overline{u^{\prime} u^{\prime}} / U^{2}$ in the blade tip region.

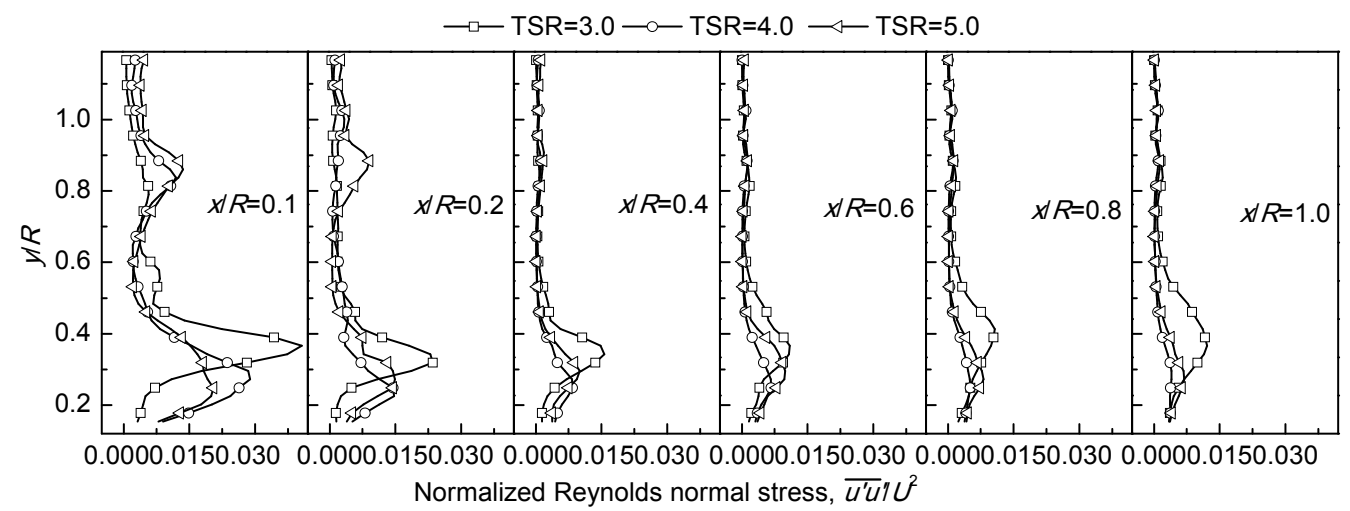

Fig. 14 Vertical profiles of the normalized Reynolds normal stress for three different tip speed ratios at six different downstream locations

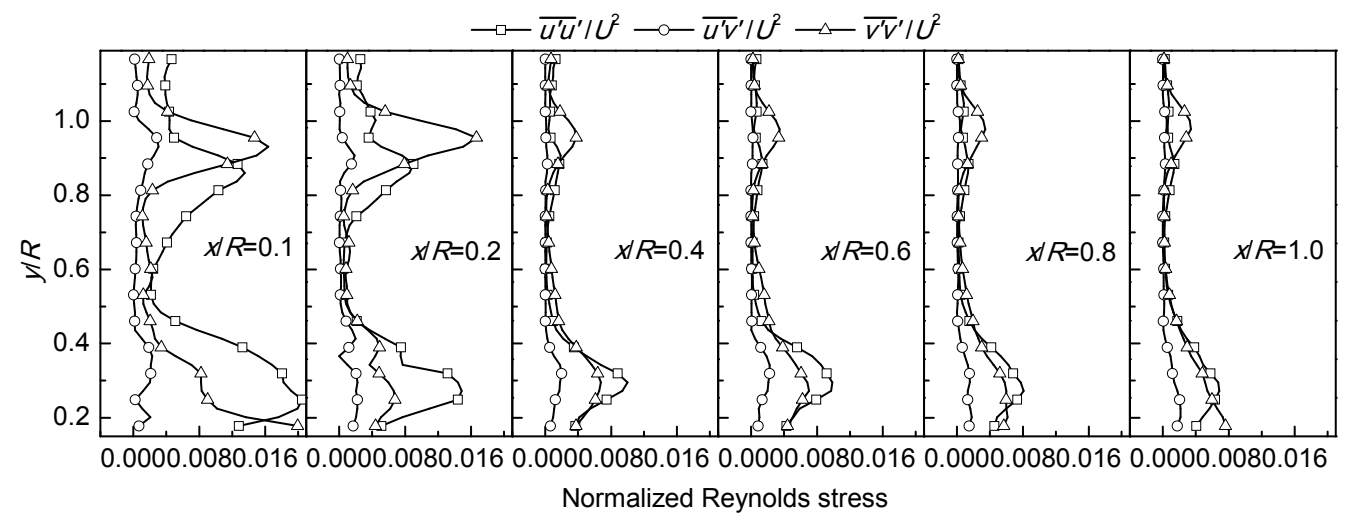

Fig. 15 Comparisons among different Reynolds stresses at six different downstream locations when the tip speed ratio is four 


\section{Conclusions}

The investigation into the flow properties of wind turbine near the wake has been carried out. The results from the present TRPIV experiment enable us to obtain the near wake detailed data such as instantaneous vorticity, average velocities, turbulence kinetic energy, and Reynolds stresses. Qualitative and quantitative analyses of the flow field results shows that: (i) the wind turbine near the wake flow field can be divided into three regions in the radial direction: velocity increased region, velocity remain unchanged region, and velocity deficit region, and the axial average velocities at different TSRs in the wake reach the inflow velocity almost at the same radial location; (ii) the radial average velocity has an increased tendency along the radial direction in the later half radial length wake region, while it decreases above the blade tip region; (iii) the turbulent kinetic energy would appear to have two peaks at approximately the $y / R=0.3$ and $y / R=0.9$ regions, and turbulence kinetic energy would meet its minimum value in the half blade radius height region along the radial direction; (iv) Reynolds shear stress is less than the Reynolds normal stresses, $\overline{u^{\prime} u^{\prime}} / U^{2}$ is larger than $\overline{u^{\prime} v^{\prime}} / U^{2}$ and $\overline{v^{\prime} v^{\prime}} / U^{2}$ in the blade root region, while $\overline{v^{\prime} v^{\prime}} / U^{2}$ is larger than $\overline{u^{\prime} v^{\prime}} / U^{2}$ and $\overline{u^{\prime} u^{\prime}} / U^{2}$ in the blade tip region.

\section{References}

Abdelsalam, A.M., Boopathi, K., Gomathinayagam, S., et al., 2014. Experimental and numerical studies on the wake behavior of a horizontal axis wind turbine. Journal of Wind Engineering and Industrial Aerodynamics, 128: 54-65. [doi:10.1016/j.jweia.2014.03.002]

Angele, K.P., Muhammad-Klingmann, B., 2006. PIV measurements in a weakly separating and reattaching turbulent boundary layer. European Journal of Mechanics-B/Fluids, 25(2):204-222. [doi:10.1016/j.euromechflu.2005.05.003]

Breton, S.P., Nilsson, K., Olivares-Espinosa, H., et al., 2014. Study of the influence of imposed turbulence on the asymptotic wake deficit in a very long line of wind turbines. Renewable Energy, 70:153-163. [doi:10.1016/j.renene. 2014.05.009]

Chen, T.Y., Liou, L.R., 2011. Blockage corrections in wind tunnel tests of small horizontal-axis wind turbines. Experimental Thermal and Fluid Science, 35(3):565-569. [doi:10.1016/j.expthermflusci.2010.12.005]

Chu, C., Chiang, P.H., 2014. Turbulence effects on the wake flow and power production of a horizontal-axis wind tur- bine. Journal of Wind Engineering and Industrial Aerodynamics, 124:82-89. [doi:10.1016/j.jweia.2013.11.001]

Hirahara, H., Hossain, M.Z., Kawahashi, M., et al., 2005. Testing basic performance of a very small wind turbine designed for multi-purposes. Renewable Energy, 30(8): 1279-1297. [doi:10.1016/j.renene.2004.10.009]

Hu, D.M., Du, Z.H., 2009. Near wake of a model horizontalaxis wind turbine. Journal of Hydrodynamics, Ser. B, 21(2):285-291. [doi:10.1016/S1001-6058(08)60147-X]

Ivanell, S., Sørensen, J.N., Mikkelsen, R., et al., 2009. Analysis of numerically generated wake structures. Wind Energy, 12(1):63-80. [doi:10.1002/we.285]

Jiang, Z.C., Doi, Y., Zhang, S.Y., 2007. Numerical investigation on the flow and power of small-sized multi-bladed straight Darrieus wind turbine. Journal of Zhejiang University-SCIENCE A, 8(9):1414-1421. [doi:10.1631/ jzus.2007.A1414]

Jin, Z., Dong, Q., Yang, Z., 2014. A stereoscopic PIV study of the effect of rime ice on the vortex structures in the wake of a wind turbine. Journal of Wind Engineering and Industrial Aerodynamics, 134:139-148. [doi:10.1016/j. jweia.2014.09.001]

Lee, H.M., Wu, Y., 2013. An experimental study of stall delay on the blade of a horizontal-axis wind turbine using tomographic particle image velocimetry. Journal of Wind Engineering and Industrial Aerodynamics, 123:56-68. [doi:10.1016/j.jweia.2013.10.005]

Lignarolo, L.E.M., Ragni, D., Krishnaswami, C., et al., 2014. Experimental analysis of the wake of a horizontal-axis wind-turbine model. Renewable Energy, 70:31-46. [doi:10. 1016/j.renene.2014.01.020]

Liu, X., Bao, Y., Li, Z., et al., 2010. Analysis of turbulence structure in the stirred tank with a deep hollow blade disc turbine by time-resolved PIV. Chinese Journal of Chemical Engineering, 18(4):588-599. [doi:10.1016/S10049541(10)60262-5]

Stafford, J., Walsh, E., Egan, V., 2012. A statistical analysis for time-averaged turbulent and fluctuating flow fields using Particle Image Velocimetry. Flow Measurement and Instrumentation, 26:1-9. [doi:10.1016/j.flowmeasinst.2012. 04.013]

Vermeer, L.J., 2001. A review of wind turbine wake research at TU Delft. ASME Wind Energy Symposium, AIAA2001-00030, p.103-113.

Vermeer, L.J., Sørensen, J.N., Crespo, A., 2003. Wind turbine wake aerodynamics. Progress in Aerospace Sciences, 39(6-7):467-510. [doi:10.1016/S0376-0421(03)00078-2]

Whale, J., Anderson, C.G., 1993. An experimental investigation of wind turbine wake using particle image velocimetry. Proceedings of the European Community Wind Energy Conference, Lubeck-Travemunde, Germany, p.457-460.

Whale, J., Papadopoulos, K.H., Anderson, C.G., et al., 1996. A study of the near wake structure of a wind turbine comparing measurements from laboratory and full-scale 
experiments. Solar Energy, 56(6):621-633. [doi:10.1016/ 0038-092X(96)00019-9]

Whale, J., Anderson, C.G., Bareiss, R., et al., 2000. An experimental and numerical study of the vortex structure in the wake of a wind turbine. Journal of Wind Engineering and Industrial Aerodynamics, 84(1):1-21. [doi:10.1016/ S0167-6105(98)00201-3]

Yang, Z., Sarkar, P., Hu, H., 2012. Visualization of the tip vortices in a wind turbine wake. Journal of Visualization, 15(1):39-44. [doi:10.1007/s12650-011-0112-z]

Zhang, W., Markfort, C.D., Porté-Agel, F., 2012. Near-wake flow structure downwind of a wind turbine in a turbulent boundary layer. Experiments in Fluids, 52(5):1219-1235. [doi:10.1007/s00348-011-1250-8]

\section{中文概要}

题 目: 基于时间分辨粒子图像测速技术的水平轴风力机 近尾迹特性的实验研究

目 的：探索风力机运行在不同尖速比下近尾迹流场的瞬 态和时均特性, 揭示风力机近尾迹场的涡量、流 速、湍流和雷诺应力演化规律, 为相关的数值模 拟提供实验对比数据支撑。
创新点: 将时间分辨粒子图像测速技术应用于风力机近尾 迹测量可以捕捉到较高时间分辨率和高精度的 流动信息, 进而揭示风力机近尾迹详细的流动 机理。

方 法: 实验在风洞里面进行, 利用时间分辨粒子图像测 速技术获得风力机瞬时流场 (图 1 3), 通过调节 风力机配套电机的负荷输出可以得到不同的运 行尖速比。对测量得到的瞬时速度的后处理可以 得到瞬时浴量以及湍动能和雷诺应力的时均值。

结 论: 1. 风力机近尾迹区域沿径向可分为速度增益区、 速度保持区和速度亏损区，不同尖速比下速度恢 复到主流速度的径向位置基本相同；2. 在后半个 半径长度区域内, 径向平均速度沿径向方向有增 加的趋势, 而在叶尖以上, 径向平均速度沿径向 减小; 3. 近尾迹湍动能在径向方向上 0.3 倍和 0.9 倍半径高度处会出现峰值, 而在 0.6 倍半径附近 位置会出现湍动能最低值; 4. 在风力机近尾迹, 雷诺剪切应力小于雷诺正应力, 在叶根附近, 轴 向雷诺正应力大于径向雷诺正应力以及雷诺剪 切应力, 而在叶尖处, 径向雷诺正应力大于轴向 雷诺正应力以及雷诺剪切应力。

关键词: 时间分辨粒子图像测速; 风力机; 尖速比; 近尾 迹; 流动特性 